\title{
Use of Basal Serum Testosterone Level as Predictor for Poor Ovarian Response in Women with Unexplained Infertility Undergoing In Vitro Fertilization Cycle: Prospective Study
}

\author{
Waleed M. Khalaf, Hayam Fathy, Sarah Safwat* \\ Faculty of Medicine, Ain Shams University, Cairo, Egypt \\ Email: *saso11_7@hotmail.com
}

How to cite this paper: Khalaf, W.M., Fathy, H. and Safwat, S. (2018) Use of Basal Serum Testosterone Level as Predictor for Poor Ovarian Response in Women with Unexplained Infertility Undergoing In Vitro Fertilization Cycle: Prospective Study. Open Journal of Obstetrics and Gynecology, 8, 1520-1531.

https://doi.org/10.4236/ojog.2018.814153

Received: November 22, 2018

Accepted: December 15, 2018

Published: December 18, 2018

Copyright $(9) 2018$ by authors and Scientific Research Publishing Inc. This work is licensed under the Creative Commons Attribution International License (CC BY 4.0).

http://creativecommons.org/licenses/by/4.0/

\section{(c) (i) Open Access}

\begin{abstract}
Background: Delayed pregnancy in women and marked increase in the numbers of older women who fail to respond to ovarian stimulation had been a significant issue. This study aims to assess the value of basal serum testosterone level as a predictor of ovarian response for induction of ovulation in women with unexplained infertility undergoing IVF (in vitro fertilization) cycle. Patients and Methods: A prospective study was conducted in Ain Shams University Maternity hospital Infertility Center during a period of time from October 2016 to June 2017. This study recruited 89 women. On day 2 or 3 of a spontaneous menstrual cycle of the included women within 3 months before fresh IVF cycle, basal hormonal (FSH, LH, estradiol, total testosterone) concentrations, AFC (antral follicle count) were performed. Using the Long-protocol for induction of ovulation, serial monitoring of ovarian response was assessed by transvaginal ultrasound. When the expected ovarian response was reached (at least three oocytes $\geq 17 \mathrm{~mm}$ ), we gave trigger dose of HCG. Ultrasound guided oocyte aspiration was performed $34-36$ hours later. Two to three days after oocyte aspiration, we transferred the embryos according to the patient's age and the condition of embryos available. Biochemical pregnancy was considered if serum B-hCG test was positive at day 14 from embryo transfer, where all the data were correlated with serum testosterone level and ovarian response as 1 ry outcome. Results: There were significant positive correlations between testosterone and LH, Prolactin, AFC, Number of oocytes \& Number of Embryos (0.014, 0.032, 0.023, 0.004, 0.033, p $<0.001$ respectively). Poor responders versus good responders as regards testosterone level $(0.81 \pm 0.47$ versus $1.08 \pm 0.45)$ Fertilized \& pregnant cases had significantly higher testosterone than non-fertilized \& non pregnant had $(1.20 \pm 0.45,0.92 \pm 0.47 \mathrm{p}$ value $0.035,0.021$ respectively). Yet, testosterone
\end{abstract}


had significant low diagnostic performance in prediction of poor response and pregnancy (AUC $0.654,0.676$ respectively), ( $\mathrm{p}$ value $0.015,0.022$ respectively). Conclusion: Basal $\mathrm{T}$ levels are helpful for predicting ovarian response, hence the dosage of gonadotropins used in induction. But it can't be used as single marker for prediction of ovarian response.

\section{Keywords}

Testosterone, Induction, Ovarian Reserve, Pregnancy

\section{Introduction}

Two to thirty percent of women undergoing induction of ovulation experience poor response which results in cycle cancellation and reduced pregnancy rate [1]. In recent years, several studies showed that response to induction in poor responders might become better with androgen supplementation. These products are cheap and available, yet their use remains controversial. This is due to lack of sufficient studies for their efficacy and safety [2] [3].

Androgen receptor mRNA is correlated with FSH receptor mRNA expression in granulose cells. Thus androgens augment FSH receptor expression in the granulose cells amplifying FSH effects on follicular growth [4] [5].

At the other extreme, androgen excess affect oocyte quality, so the current study excluded patients with hyperandrogenemia as in PCOS (polycystic ovarian syndrome) [6].

A previous study by Guo et al. suggested that basal testosterone, instead of DHEAS concentration, couldn't be used as single predictor, for POR (poor ovarian reserve). However multifactors as age, AFC, basal FSH, basal FSH/LH and basal testosterone are better predictors for POR and clinical pregnancy than AFC alone. They also observed that as testosterone levels increase, total gonadotropins dosage decrease while numbers of oocytes retrieved, cleavage-stage embryos, frozen embryos and pregnancy rates all increase significantly [7].

Assessment of serum androgen levels prior to controlled ovarian stimulation might be useful to predict the ovarian response and, thus adjust the starting dose of exogenous gonadotrophins or even pretreatment with transdermal testosterone before ovarian stimulation may be a useful approach for women known to have poor ovarian response.

This study aims to assess the value of basal serum testosterone level as a predictor of ovarian response for induction of ovulation in women undergoing IVF cycle.

\section{Patients and Methods}

A prospective study was conducted in Ain Shams University Maternity hospital infertility center in the period from October 2016 and June 2017. This study recruited 89 women with unexplained infertility. Patients were between 21 and 35 years of age, all patients complained of primary or secondary infertility in spite of conti- 
nuous marital life for at least two years after marriage, all patients were ovulatory and had regular menstrual cycles, BMI: 20 - 30, normal pelvic transvaginal ultrasonography concerning exclusion of pelvic pathology, normal hysterosalpingogram (confirming tubal patency and showing absence of uterine anomalies or filling defects, or tubal anomalies as hydrosalpinx), normal hormonal profile regarding estradiol, FSH (Day $2<10 \mathrm{mIU} / \mathrm{ml}$ ), LH (Day $2<8 \mathrm{mIU} / \mathrm{ml}$ ), TSH $(0.5-4.5 \mathrm{mIU} / \mathrm{L}$ ), serum prolactin ( $2-29 \mathrm{ng} / \mathrm{ml})$.All husbands of the selected patients showed normal semen analysis parameters. Women with endocrinal problems such as DM, thyroid disorders or hyperprolactinemia were excluded. Women having AFC $<5$ follicles (Antral Follicular Count in both ovaries) were also excluded.

Ethics: The study was approved from the Ethical Committee of the Department of Obstetrics and Gynecology, Faculty of Medicine, Ain Shams University. Written informed consents were obtained from all participants.

All participants were subjected to careful history taking and clinical examination including BMI to ensure fulfillment of selection criteria. Completion of the series of investigations to fulfill the selection criteria on day 2 or 3 of a spontaneous menstrual cycle within 3 months before fresh IVF cycle, a blood sample was taken in the morning to evaluate basal hormonal (FSH, LH, estradiol, total testosterone): Complete Blood Picture, Thyroid Profile, prolactin, AMH (antimullerian hormone). On the same day, trans-vaginal sonography was performed to obtain AFC (the follicles visualized and counted were $2-10 \mathrm{~mm}$ in size, and the numbers of follicles in both ovaries were added to obtain the total AFC) [8], at the same time will be used to identify uterine fibroids, PCO, polyps. Recent hysterosalpingogram \& semen analysis of husband should be availabe.

\section{Using the Long-protocol for induction of ovulation:}

This study used controlled-release of long-acting GnRH analogue (Decapeptyl $^{\circledR} ; 0.1 \mathrm{mg}$ /ampoule Tryptorelin, Ferring, Germany) daily from mid-luteal day of the previous cycle till the day of hCG administration. When a satisfactory pituitary desensitization is achieved (Estradiol level below $40 \mathrm{pg} / \mathrm{ml}$ ), human menopausal gonadotrophins (Menogon ${ }^{\circledR}$; 75 mg/ampoule HMG, Ferring GmbH Wittland 11, $1 \mathrm{Kiel}$, Germany) was given to women. The starting dosage was also prescribed on an individual basis according to the patient's basal status. Serial monitoring of ovarian response was assessed by transvaginal ultrasound. A trigger dose of B-hCG (Choriomon ${ }^{\circledR}$; 5000 IU/ampoule $\{2$ ampoules\}, IBSA, Egypt) was administered when we reached the expected ovarian response (at least three oocytes $\geq 17 \mathrm{~mm}$ ). Ultrasound guided oocyte aspiration was performed $34-36 \mathrm{~h}$ later. Standard laboratory protocols were followed. Two to three days after oocyte aspiration, transferal of embryos according to the patient's age and the condition of embryos available. As a rule, and if available, two embryos were transferred. This was followed by luteal phase support using Progesterone (Prontogest $^{\circledR} ; 400 \mathrm{mg}$ /suppository, Marcyrl, Egypt) starting on the day of oocyte retrieval and continuing either up to menstruation, or if the patient became pregnant, at least the first 8 weeks of pregnancy. 
Biochemical pregnancy was considered if serum B-hCG test was positive at day 14 from embryo transfer, where all the data were correlated with serum testosterone level, with ovarian responders(the $1^{\text {ry }}$ outcome) and pregnancy outcome (the $2^{\text {ry }}$ outcome).

Hormonal Assay:

All blood samples were immediately processed to separate serum. Serum samples were stored at $-20^{\circ} \mathrm{C}$ and hormonal assays were performed in the Endocrine Laboratory of Ain Shams University Hospital, Cairo, Egypt using Enzyme-Linked Immunosorbent Assay for the Quantitative Determination in $\mathrm{Hu}$ man Serum (ELISA Technique) by kits named Immunospec Quantitative Assay (Immunospec Corporation, 7018 Owensmouth Ave. Suite 103, Canoga Park, CA, 91303) according to the manufacture instructions to Testosterone, FSH, LH and Estradiol with analytical sensitivity is $<0.1 \mathrm{ng} / \mathrm{ml}, 2.5 \mathrm{mIU} / \mathrm{ml}, 2 \mathrm{mIU} / \mathrm{ml}, 5$ $\mathrm{pg} / \mathrm{ml}$ respectively.

Statistical methods:

The collected data were coded, tabulated, and statistically analyzed using IBM SPSS statistics (Statistical Package for Social Sciences) software version 22.0, IBM Corp., Chicago, USA, 2013.

Descriptive statistics were done for quantitative data as minimum\& maximum of the range as well as mean $\pm \mathrm{SD}$ (standard deviation) for quantitative parametric data, while it was done for qualitative data as number and percentage.

Inferential analyses were done for quantitative variables using independent t-test in cases of two independent groups with parametric data. In qualitative data, inferential analyses for independent variables were done using Chi square test for differences between proportions. While correlations were done using Pearson correlation for numerical parametric data. Logistic regression model was used to find out independent factors affecting response. The level of significance was taken at $\mathrm{P}$ value $<0.050$ is significant, otherwise is non-significant.

\section{Results}

1) Basal characteristics of the studied women were listed in Table 1.

2) Fertilization characteristics of the studied women. The number of fertilized women in the study 77 out of 89 which represents $88.5 \%$ yielding mean number of embryos of 5.5 (Table 2).

3) Pregnancy rate among the studied women was $20.2 \%$ (18 out of 89) (Table 3).

\section{Correlation between Testosterone and Other Variables}

There were significant positive correlations between testosterone and LH, Prolactin, AFC, Number of oocytes \& Number of Embryos (P value 0.014, 0.032, $0.023,0.033,<0.001$ respectively) (Table 4 ).

\section{Relation of Fertilization with Testosterone (nmol/L)}

Fertilized cases had significantly higher testosterone than non-fertilized had ( $\mathrm{P}$ value 0.002 ) (Table 5). 
Table 1. Basal characteristics of the studied women.

\begin{tabular}{ccc}
\hline Characteristics & Mean $\pm \mathrm{SD}$ & Range \\
\hline Age (years) & $28.5 \pm 3.6$ & $21.0-35.0$ \\
BMI $\left(\mathrm{kg} / \mathrm{m}^{2}\right)$ & $27.9 \pm 1.2$ & $25.1-31.5$ \\
Parity & $4.6 \pm 1.4$ & $2.0-8.0$ \\
FSH (mIU/mL) & $1.0 \pm 0.8$ & $0.0-2.0$ \\
LH (mIU/mL) & $7.45 \pm 2.1$ & $4.2-10.7$ \\
FSH/LH ratio & $5.0 \pm 1.3$ & $2.1-8.3$ \\
Prolactin $(\mathrm{ng} / \mathrm{mL})$ & $1.9 \pm 0.9$ & $0.7-5.6$ \\
E2 (pg/mL) & $19.3 \pm 4.6$ & $6.8-30.4$ \\
TSH (mIU/L) & $53.2 \pm 13.5$ & $22.0-85.4$ \\
Testosterone $(\mathrm{nmol} / \mathrm{L})$ & $3.1 \pm 0.2$ & $2.6-3.6$ \\
\hline
\end{tabular}

Total $=89$.

Table 2. Fertilization characteristics of the studied women.

\begin{tabular}{ccc}
\hline Characteristics & Mean \pm SD & Range \\
\hline Number of embryos & $5.5 \pm 2.9$ & $0.0-13.0$ \\
\hline & $\mathrm{N}$ & $\%$ \\
\hline Fertilization & 77 & 88.5 \\
\hline
\end{tabular}

total $=89$.

Table 3. Pregnancy among the studied women.

\begin{tabular}{ccc}
\hline Characteristics & N & $\%$ \\
\hline Among all cases $($ Total $=89)$ & 18 & 20.2 \\
Among fertilized cases $($ Total $=77)$ & 18 & 23.4 \\
\hline
\end{tabular}

Table 4. Correlation between testosterone and other variables.

\begin{tabular}{ccc}
\hline Variables & $\mathrm{R}$ & $\mathrm{P}$ \\
\hline Age & -0.209 & $0.049^{\star}$ \\
BMI & -0.228 & $0.032^{\star}$ \\
Duration of infertility & -0.104 & 0.333 \\
Parity & 0.112 & 0.294 \\
FSH & -0.252 & $0.017^{*}$ \\
LH & 0.260 & $0.014^{\star}$ \\
FSH/LH ratio & -0.327 & $0.002^{\star}$ \\
Prolactin & 0.228 & $0.032^{\star}$ \\
E2 & -0.250 & $0.018^{*}$ \\
TSH & -0.220 & $0.038^{*}$ \\
AFC & 0.240 & $0.023^{*}$ \\
Duration of ovarian stimulation & -0.307 & $0.003^{*}$ \\
HMG dose & -0.299 & $0.004^{*}$ \\
Number of oocytes & 0.226 & $0.033^{\star}$ \\
Number of Embryos & 0.423 & $<0.001^{*}$ \\
\hline
\end{tabular}

Pearson correlation, ${ }^{\star}$ Significant. 


\section{Comparison between Poor and Good Responses regarding Basal Characteristics}

Poor responders versus good responders as regards testosterone level $(0.81 \pm$ 0.47 versus $1.08 \pm 0.45$ ). Poor responders had significantly higher age and BMI than good responders had. Poor responders had significantly higher FSH, FSH/LH ratio, E2 and TSH and significantly lower LH, prolactin and Testosterone than good responders had (Table 6).

\section{Comparison between Pregnancy Conditions regarding Basal Characteristics}

Pregnant cases had significantly higher testosterone than non pregnant had $(0.92$ $\pm 0.47 \mathrm{p}$ value, 0.026 ) according to Table 7 .

\section{Diagnostic Performance of Testosterone in Prediction of Outcomes}

Testosterone had significant low diagnostic performance in prediction of poor response and pregnancy, so it can't be used as marker alone for ovarian response (Table 8 and Figure 1).

Table 5. Relation of fertilization with testosterone ( $\mathrm{nmol} / \mathrm{L})$.

\begin{tabular}{ccccc}
\hline & & N & Mean \pm SD & P value \\
\hline \multirow{2}{*}{ Fertilization } & Fertilized & 77 & $1.04 \pm 0.46$ & $0.002^{*}$ \\
& Not & 12 & $0.56 \pm 0.34$ & \\
\hline
\end{tabular}

${ }^{\wedge}$ Independent t-test, ${ }^{*}$ significant.

Table 6. Comparison between poor and good responses regarding basal characteristics.

\begin{tabular}{|c|c|c|c|}
\hline Characteristics & Poor $(\mathrm{N}=34)$ & Good $(\mathrm{N}=55)$ & $\mathrm{P}$ \\
\hline Age (years) & $31.3 \pm 3.0$ & $28.4 \pm 3.5$ & ${ }^{\wedge}<0.001^{*}$ \\
\hline $\operatorname{BMI}\left(\mathrm{kg} / \mathrm{m}^{2}\right)$ & $28.5 \pm 1.1$ & $27.6 \pm 1.2$ & ${ }^{\wedge}<0.001^{*}$ \\
\hline Duration of infertility (years) & $4.9 \pm 1.3$ & $4.5 \pm 1.4$ & ${ }^{\wedge} 0.153$ \\
\hline Parity & $0.9 \pm 0.8$ & $1.0 \pm 0.8$ & ${ }^{\wedge} 0.516$ \\
\hline $\mathrm{FSH}(\mathrm{mIU} / \mathrm{mL})$ & $9.4 \pm 2.1$ & $8.3 \pm 1.9$ & $0.019^{*}$ \\
\hline LH (mIU/mL) & $4.6 \pm 1.3$ & $5.3 \pm 1.3$ & $0.020^{*}$ \\
\hline $\mathrm{FSH} / \mathrm{LH}$ ratio & $2.22=1.0$ & $1.7 \pm 0.7$ & $0.004^{*}$ \\
\hline Prolactin (ng/mL) & $17.6 \pm 5.3$ & $20.3 \pm 3.7$ & $0.007^{\star}$ \\
\hline $\mathrm{E} 2(\mathrm{pg} / \mathrm{mL})$ & $58.2 \pm 15.0$ & $50.1 \pm 11.5$ & $0.005^{\star}$ \\
\hline TSH (mIU/L) & $3.2 \pm 0.2$ & $3.0 \pm 0.2$ & $<0.001^{\star}$ \\
\hline Testosterone (nmol/L) & $0.81 \pm 0.47$ & $1.08 \pm 0.45$ & $0.008^{\star}$ \\
\hline \multicolumn{4}{|l|}{ Fertilization } \\
\hline Embryo number & $4.7 \pm 2.6$ & $6.0 \pm 3.0$ & $0.044^{*}$ \\
\hline Fertilization & $25(78.1 \%)$ & $52(94.5 \%)$ & $0.021^{\star}$ \\
\hline Pregnancy & $3(8.8 \%)$ & $15(27.3 \%)$ & $0.035^{\star}$ \\
\hline
\end{tabular}

${ }^{\wedge}$ Independent t-test, \#Chi square test, *Significant. 
Table 7. Comparison between pregnancy conditions regarding basal characteristics.

\begin{tabular}{cccc}
\hline Characteristics & Pregnant $(\mathrm{N}=18)$ & Not $(\mathrm{N}=71)$ & $\mathrm{P}$ \\
\hline Age (years) & $28.1 \pm 3.3$ & $29.8 \pm 3.6$ & 0.073 \\
BMI $\left(\mathrm{kg} / \mathrm{m}^{2}\right)$ & $28.0 \pm 1.0$ & $27.9 \pm 1.3$ & 0.870 \\
Duration of infertility (years) & $4.6 \pm 1.6$ & $4.6 \pm 1.3$ & 0.830 \\
Parity & $1.3 \pm 0.8$ & $0.9 \pm 0.8$ & 0.063 \\
FSH (mIU/mL) & $8.1 \pm 2.2$ & $8.9 \pm 2.0$ & 0.149 \\
LH (mIU/mL) & $5.5 \pm 1.5$ & $4.9 \pm 1.3$ & 0.113 \\
FSH/LH ratio & $1.6 \pm 0.8$ & $2.0 \pm 0.9$ & 0.165 \\
Prolactin (ng/mL) & $19.5 \pm 2.9$ & $19.2 \pm 4.9$ & 0.816 \\
E2 (pg/mL) & $50.3 \pm 10.5$ & $53.9 \pm 14.1$ & 0.312 \\
TSH (mIU/L) & $3.0 \pm 0.2$ & $3.1 \pm 0.2$ & 0.324 \\
Testosterone (nmol/L) & $1.20 \pm 0.45$ & $0.92 \pm 0.47$ & $0.026^{*}$ \\
\hline
\end{tabular}

${ }^{\wedge}$ Independent t-test, \#Chi square test, ${ }^{\star}$ Significant.

Table 8. Diagnostic performance of Testosterone in prediction of outcomes.

\begin{tabular}{ccccc}
\hline Condition & AUC & SE & P & $95 \%$ CI \\
\hline Poor response & 0.654 & 0.061 & $0.015^{\star}$ & $0.535-0.773$ \\
Pregnancy & 0.676 & 0.071 & $0.022^{\star}$ & $0.538-0.815$ \\
\hline
\end{tabular}

AUC: Area under curve, SE: Standard error, CI: Confidence interval, ^Significant.

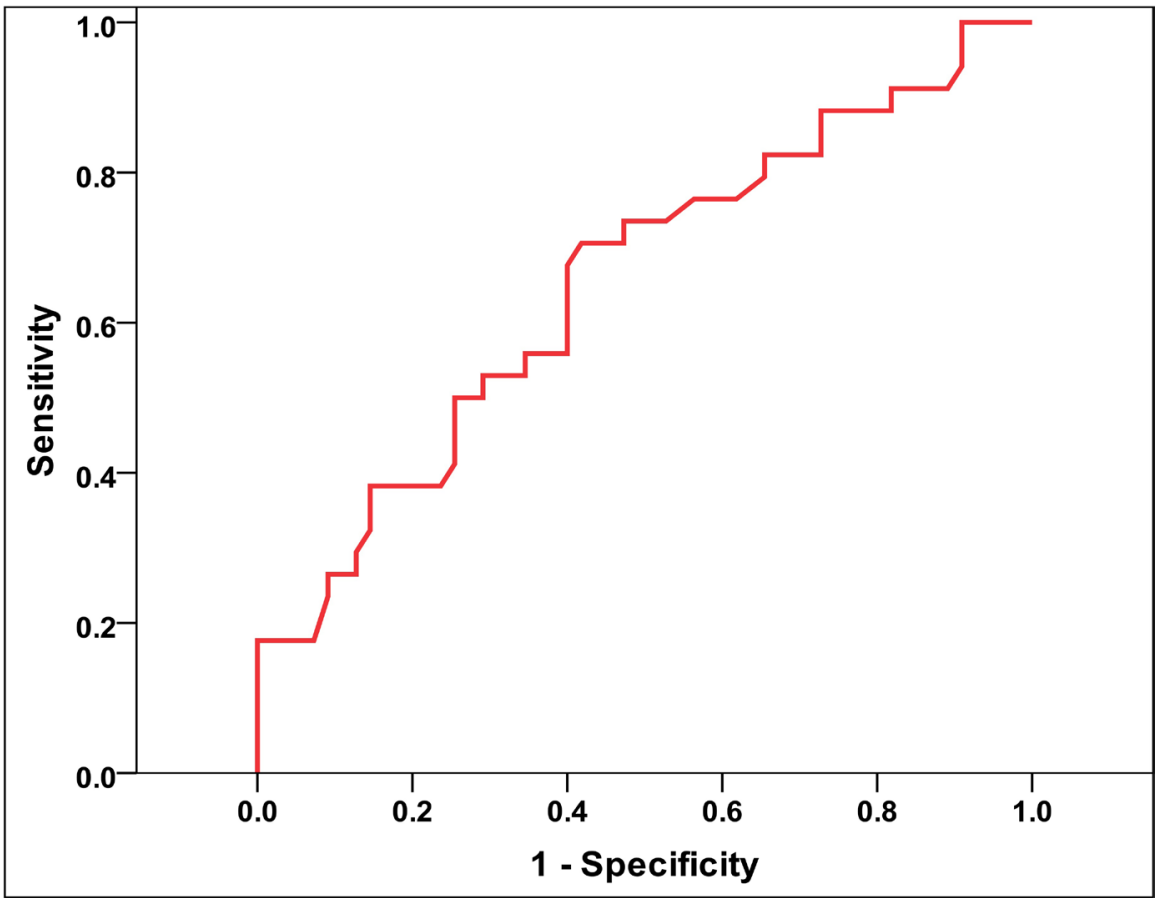

Figure 1. ROC curve for Testosterone in prediction of poor outcome showing low diagnostic performance of testosterone in prediction of poor response and pregnancy. 


\section{Discussion}

Several reviews have showed the predictive value of many tests for ovarian reserve, antral follicle count (AFC) and anti-Mullerianhormone (AMH) were the best. However, even the best marker had a false positive rate of $10 \%-20 \%$, might falsely prevent these women from undergoing IVF [9] [10].

In women undergoing IVF cycle who attended Ain shams university maternity hospital during October 2015 to June 2016, this study compared basal serum testosterone level in relation to number of follicles in both ovaries(AFC),number of actual oocyte retrieved, number of embryos fertilized, positive pregnancy test to predict PORs (poor ovarian responders).

The current study suggested that serum testosterone level may help in prediction of the type of response for induction of ovulation in women undergoing IVF cycle.

89 women underwent the ovarian stimulation protocol \& oocyte aspiration, where 55 women (61.8\%) have been good responders, while 34 women (38.2\%) have been poor responders. Testosterone level among PORs was $0.81 \pm 0.47$ $\mathrm{nmol} / \mathrm{L}$ where it was $1.08 \pm 0.45 \mathrm{nmol} / \mathrm{L}$ among good responders. Also its level among pregnant women was $1.20 \pm 0.45 \mathrm{nmol} / \mathrm{L}$, while among non-pregnant women was $0.92 \pm 0.47 \mathrm{nmol} / \mathrm{L}$.

Correlation between testosterone and other variables showed that: There were significant positive correlations between testosterone and BMI, LH, Prolactin, AFC, Number of oocytes \& Number of Embryos, while there were significant negative correlations between testosterone and age, FSH, FSH/LH ratio, E2, TSH, Duration of ovarian stimulation \& HMG dose.

These results were concordant with Jing Guo et al. (2014) who found that basal $\mathrm{T}$ positively related to body mass index (BMI), AFC, numbers of oocytes retrieved, mature oocytes and pregnancy outcome, but negatively with age, basal FSH/LH, peak E2, and total gonadotropins dosage. Also these results were confirmed by Bo Sun et al. (2014), with the exception of falling of basal E2 levels in the positively correlated parameters. That may be due to different magnitude of populations between the studies or the applied tense whether prospectively or retrospectively [6] [11].

Also these results went with that of Fraterelli and Gerber (2006) and Qin et al., 2011) confirming the above data. Colakoglu (1986) found that Testosterone and DHEAs level decline with age, which may be related to POR predicted by FSH and age [9] [12] [13].

As regards relation of fertilization with testosterone level showed that Fertilized cases had significantly higher testosterone than non-fertilized had.

Comparison between poor and good responses regarding demographic characteristics and hormonal characteristics showed that poor responders had significantly higher age, BMI, FSH, FSH/LH ratio, E2 and TSH than good responders had. Poor responders had significantly lower $\mathrm{LH}$, prolactin and Testosterone than good responders had.

This is in consistent with Guo, J. et al. 2014, poor ovarian responders were 
significantly older than normal ovarian responders $(\mathrm{P}<0.001)$, with higher body mass index (BMI; $\mathrm{P}=0.001)$, basal FSH $(\mathrm{P}<0.001)$, FSH/LH $(\mathrm{P}<0.001)$ and estradiol $(\mathrm{P}<0.001)$ and lower AFC $(\mathrm{P}<0.001)$, mean ovarian volume $(\mathrm{P}<0.001)$ and testosterone $(P=0.022)$. Significantly higher total gonadotropins dosage was consumed and lower peak estradiol, number of mature oocytes, fertilization rate, cleavage rate, implantation rate and clinical pregnancy rate (all $\mathrm{P}<0.001$ ) were achieved by poor ovarian responders. Jayaprakasan et al., 2009 confirmed the above data [6] [14].

On the other hand, Bo Sun et al. (2014) found that the good responder women had more oocytes retrieved, good quality embryos, and embryos cryopreserved. However, the pregnancy outcomes were not significantly different between the two groups [11].

Comparison between pregnancy conditions regarding demographic characteristics showed no significant difference. While comparison between pregnancy conditions regarding basal hormonal characteristics showed that pregnant women had significantly higher testosterone (T) than non-pregnant women had.

Also comparison between pregnancy conditions regarding stimulation, retrieval and fertilization showed that pregnant women had significantly higher good response, oocyte and embryo numbers and significantly lower duration and dose than non-pregnant women had.

Also Jing Guo et al. (2014) observed that Testosterone levels of pregnant women were significantly higher than those who were not (1.3 \pm 0.57 vs $1.23 \pm$ 0.6 nmolL; $P=0.026$ ) [6].

Testosterone had significant low diagnostic performance in prediction of poor response and pregnancy, in other words can't be used as a marker alone for prediction of ovarian response.

Yingying Qin's group (2011) suggested that basal T level was a predictor for ovarian response and pregnancy outcome in women with diminished ovarian reserve; but not in those with normal serum FSH. Also, Fratharelli and Peterson's study (2004) showed that women with Day 3 testosterone level lesser than $20 \mathrm{ng} / \mathrm{dl}$ were five time less likely to achieve pregnancy approved by Fouany and Sharara, 2013.This may be attributed to the theory of ovarian aging suggested by Glechier and Barad (2011) [9] [12] [15] [16].

Disagreement with this opinion Frattarelli and Gerber (2006) adopted the negative relation between basal Testosterone level and pregnancy outcome. Many patients who have poor gonadotropin responsiveness and low-quality oocytes and embryos having normal screening results Thus, the tests for diminished ovarian reserve are specific but not sensitive [9].

Another study performed by Juan Balaschfound that pretreatment of poorresponder patients to controlled ovarian stimulation with androgens for assisted reproduction, who have normal basal FSH concentrations, $80 \%$ showed an fivefold increase in the number of recruited follicles, received two or three embryos 
and achieved a clinical pregnancy rate of $30 \%$ per oocyte retrieval. There were $20 \%$ cancelled cycles [17].

Most studies of serum $\mathrm{T}$ levels in infertile women have focused on women with irregular cycles and PCOS. In this study, women with irregular menstrual cycles or a known diagnosis of PCOS were excluded.

The multivariate model composed of age, AFC, basal FSH, basal FSH/LH and basal testosterone performed better than AFC for predicting both POR and pregnancy outcome. This might be meaningful for most of reproductive medicine, where AMH measurement is costly and not in routine use, however testosterone is available, cheap \& can be used as preinduction treatment to enhance ovarian response in those with previous poor response.

Identification of women at increased risk for POR prior to IVF could be useful, help to modify the gonadotropins dose for induction in order to maximize ovarian response [18].

\section{Limitation of the Study}

Measuring only basal serum T level, which is an ovarian androgen,. DHEA and other adrenal androgens are also very important.

Another limitation of study, it didn't study the role and the effect of giving androgens to those of low levels of testosterone and POR \& study the change in ovarian response, another drop out that it didn't mention the relation between the degree of severity of low testosterone level and ovarian response. Another research may be needed to assess the role of basal testosterone level and preinduction treatment with androgens in those with poor ovarian reserve according to bologna criteria two of those criteria: Advanced maternal age ( $\geq 40$ years) or any other risk factor for POR, previous POR ( $\leq$ three oocytes with a conventional stimulation protocol).An abnormal ovarian reserve tests (i.e. AFC, 5 - 7 follicles or AMH, $0.5-1.1 \mathrm{ng} / \mathrm{ml})$.

Also this research not including obese women and relation of obesity to basal testosterone level and if preinduction treatment with androgens can be of benefit for those obese infertile women with poor ovarian response. This question need to be put in mind in future studies.

\section{Conclusion}

Basal $\mathrm{T}$ levels are helpful for predicting ovarian response, hence the dosage of gonadotropins used in induction. Yet, testosterone had significant low diagnostic performance in prediction of poor response and pregnancy, so can't be used as single marker for prediction of ovarian response.

\section{Acknowledgements}

Special thanks go to the patients and their families for the great support of our work. Also, the authors appreciate the support from infertility center, ultrasound unit\& clinical pathology staff, laboratory staff of Ain Shams University Materni- 
ty Hospital for the outstanding support during the entire study.

\section{Compliance with Ethical Standards}

\section{Disclosure Statement}

No potential conflict of interest was reported by the authors.

\section{Funding}

This research did not receive any specific grant from funding agencies in the public, commercial, or not-for-profit sectors.

\section{References}

[1] Hendriks, D.J., Mol, B.W., Bancsi, L.F., Te, V.E. and Broekmans, F.J. (2005) Antral Follicle Count in the Prediction of Poor Ovarian Response and Pregnancy after In Vitro Fertilization: A Meta-Analysis and Comparison with Basal Follicle-Stimulating Hormone Level. Fertility and Sterility, 83, 291-301.

https://doi.org/10.1016/j.fertnstert.2004.10.011

[2] Kim, C.H., Howles, C.M. and Lee, H.A. (2011) The Effect of Transdermal Testosterone Gel Pretreatment on Controlled Ovarian Stimulation and IVF Outcome in Low Responders. Fertility and Sterility, 95, 679-683. https://doi.org/10.1016/j.fertnstert.2010.07.1077

[3] Nagels, H.E., Rishworth, J.R., Siristatidis, C.S., Kroon, B., et al. (2012) Androgens (Dehydroepiandrosterone or Testosterone) in Women Undergoing Assisted Reproduction (Protocol), Copyright (C) 2012 The Cochrane Collaboration. John Wiley \& Sons, Ltd.

[4] Weil, S., Vendola, K., Zhou, J. and Bondy, C.A. (1999) Androgen and Follicle-Stimulating Hormone Interactions in Primate Ovarian Follicle Development. The Journal of Clinical Endocrinology \& Metabolism, 84, 2951-2956. https://doi.org/10.1210/jcem.84.8.5929

[5] Hugues, J.N. and Durnerin, I.C. (2005) Impact of Androgens on Fertility-Physiological, Clinical and Therapeutic Aspects. Reproductive BioMedicine Online, 11, 570-580. https://doi.org/10.1016/S1472-6483(10)61165-0

[6] Guo, J., Zhang, Q., Li, Y., Huang, J., Wang, W., Huang, L., Zhao, X. and Yang, D. (2014) Predictive Value of Androgens and Multivariate Model for Poor Ovarian Response. Reproductive BioMedicine Online, 28, 723-732. https://doi.org/10.1016/j.rbmo.2014.02.009

[7] Nielsen, M.E., Rasmussen, I.A., Kristensen, S.G., Christensen, S.T., Mollgard, K., Wreford, A.E., Byskov, A.G. and Yding, A.C. (2011) In Human Granulosa Cells from Small Antral Follicles, Androgen Receptor mRNA and Androgen Levels in Follicular Fluid Correlate with FSH Receptor mRNA. Molecular Human Reproduction, 17, 63-70. https://doi.org/10.1093/molehr/gaq073

[8] Qin, Y., Zhao, Z., Sun, M., Geng, L., Che, L. and Chen, Z. (2011) Association of Basal Serum Testosterone Levels with Ovarian Response and In Vitro Fertilization Outcome. Reproductive Biology and Endocrinology, 9, 9. https://doi.org/10.1186/1477-7827-9-9

[9] Broekmans, F.J., de Ziegler, D., Howles, C.M., Gougeon, A., Trew, G. and Olivennes, F. (2010) The Antral Follicle Count: Practical Recommendations for Better Standardization. Fertility and Sterility, 94, 1044-1051. 
https://doi.org/10.1016/j.fertnstert.2009.04.040

[10] Colakoglu, M. (1986) The Effect of Dehydroepiandrosterone Sulfate Prolactin and Testosterone Hormones in Female Fertility and Hirsutism. Clinical and Experimental Obstetrics and Gynecology, 13, 32-34.

[11] Ferraretti, A.P., La Marca, A., Fauser, B.C., Tarlatzis, B., Nargund, G. and Gianaroli, L. (2011) ESHRE Consensuson the Definition of "Poor Response" to Ovarian Stimulation for In Vitro Fertilization: The Bologna Criteria. Human Reproduction, 26, 1616-1624. https://doi.org/10.1093/humrep/der092

[12] La Marca, A., Sighinolfi, G., Radi, D., Argento, C., Baraldi, E., Artenisio, A.C., Stabile, G. and Volpe, A. (2010) Anti-Mullerian Hormone (AMH) as a Predictive Marker in Assisted Reproductive Technology (ART). Human Reproduction Update, 16, 113-130. https://doi.org/10.1093/humupd/dmp036

[13] Jayaprakasan, K., Al-Hasie, H., Jayaprakasan, R., Campbell, B., Hopkisson, J., Johnson, I. and Raine-Fenning, N. (2009) The Three-Dimensional Ultrasonographic Ovarian Vascularity of Women Developing Poor Ovarian Response during Assisted Reproduction Treatment and Its Predictive Value. Fertility and Sterility, 92, 1862-1869. https://doi.org/10.1016/j.fertnstert.2008.09.031

[14] Fouany, M.R. and Sharara, F.I. (2013) Is There a Role for DHEA Supplementation in Women with Diminished Ovarian Reserve? Journal of Assisted Reproduction and Genetics, 30, 1239-1244. https://doi.org/10.1007/s10815-013-0018-x

[15] Gleicher, N. and Barad, D.H. (2011) Dehydroepiandrosterone (DHEA) Supplementation in Diminished Ovarian Reserve (DOR). Reproductive Biology and Endocrinology, 9.

[16] Balasch, J., Fábregues, F., Peñarrubia, J., Carmona, F., Casamitjana, R., Creus, M., Manau, D., Casals, G. and Vanrell, J.A. (2006) Pretreatment with Transdermal Testosterone May Improve Ovarian Response to Gonadotrophins in Poor-Responder IVF Patients with Normal Basal Concentrations of FSH. Human Reproduction, 21, 1884-1893. https://doi.org/10.1093/humrep/del052

[17] Sun, B., Wang, F., Sun, J., Yu, W.Z. and Sun, Y.P. (2014) Basal Serum Testosterone Levels Correlate with Ovarian Response But Do Not Predict Pregnancy Outcome in Non-PCOS Women Undergoing IVF. Journal of Assisted Reproduction and Genetics, 31, 829-835. https://doi.org/10.1007/s10815-014-0246-8

[18] Klinkert, E.R., Broekmans, F.J., Looman, C.W., Habbema, J.D. and Te, V.E. (2005) Expected Poor Responders on the Basis of an Antral Follicle Count Do Not Benefit from a Higher Starting Dose of Gonadotrophins in IVF Treatment: A Randomized Controlled Trial. Human Reproduction, 20, 611-615.

https://doi.org/10.1093/humrep/deh663 